Revue internationale P.M.E.

Économie et gestion de la petite et moyenne entreprise

\title{
Usages du concept de cognition dans les travaux de recherche sur l'entrepreneuriat
}

\section{Pierre Cossette}

Volume 23, numéro 1, 2010

URI : https://id.erudit.org/iderudit/045743ar

DOI : https://doi.org/10.7202/045743ar

Aller au sommaire du numéro

\section{Éditeur(s)}

Presses de l'Université du Québec

ISSN

0776-5436 (imprimé)

1918-9699 (numérique)

Découvrir la revue

Citer cet article

Cossette, P. (2010). Usages du concept de cognition dans les travaux de recherche sur l'entrepreneuriat. Revue internationale P.M.E., 23(1), 9-32. https://doi.org/10.7202/045743ar

\section{Résumé de l'article}

Plusieurs chercheurs reconnaissent aujourd'hui l'importance de la dimension cognitive dans le renouvellement des connaissances sur l'entrepreneuriat. Cependant, l'emploi que les chercheurs font du vocabulaire cognitif, en particulier du concept même de cognition, est encore peu documenté. Pourtant, une meilleure connaissance des usages que les chercheurs font de ce concept serait susceptible de mieux faire ressortir les intérêts des chercheurs concernés, de stimuler chez eux la réflexion et la discussion d'ordre théorique associée à l'emploi du concept de cognition en entrepreneuriat ainsi qu'à mieux positionner leurs propres travaux de recherche. La présente étude vise donc à répondre à la question suivante : Quels usages les chercheurs font-ils du concept de cognition dans leurs travaux sur l'entrepreneuriat ? De façon plus précise, l'objectif de cette recherche est de répertorier et caractériser l'ensemble des expressions à l'intérieur desquelles figure le concept de cognition dans la littérature savante sur l'entrepreneuriat. Pour parvenir à faire ainsi le point sur l'utilisation des expressions cognitives par les chercheurs en entrepreneuriat, nous avons consulté la base de données ABI/INFORM Global. En nous appuyant sur les informations obtenues, nous avons pu décrire de manière à la fois globale et précise ce à quoi s'intéressent les chercheurs en entrepreneuriat qui font appel au concept de cognition. Les informations analysées indiquent d'abord que les concepts d'entrepreneuriat et de cognition se trouvent simultanément dans 134 titres ou résumés d'articles publiés depuis 1993 et que leur présence conjointe va en s’accentuant. Sur le plan théorique, ces informations ont permis d'élaborer un cadre de référence spécifique aux chercheurs en entrepreneuriat qui font appel au concept de cognition. Très concrètement, les résultats montrent que les expressions contenant le mot cognition ou un de ses dérivés renvoient souvent à une grande orientation sur le plan théorique ou épistémologique (p. ex., cognitive perspective), parfois à des concepts généraux (p. ex., cognitive factors, cognitive dimension) et, très fréquemment, à des objets particuliers laissant voir ce que la cognition caractérise (p. ex., des systèmes référentiels, des outils, des aptitudes) ou encore comment elle est elle-même caractérisée (p. ex., par son niveau individuel ou collectif, par sa complexité, par sa cohérence). Les limites de cette classification en catégories et sous-catégories sont discutées et différentes voies de recherche sont envisagées.
Ce document est protégé par la loi sur le droit d'auteur. L'utilisation des services d'Érudit (y compris la reproduction) est assujettie à sa politique d'utilisation que vous pouvez consulter en ligne.

https://apropos.erudit.org/fr/usagers/politique-dutilisation/ 


\title{
Usages du concept de cognition dans les travaux de recherche sur l'entrepreneuriat'
}

Pierre COSSETTE

École des sciences de la gestion

Université du Québec à Montréal

MOTS CLÉS

\section{Cognition - Entrepreneuriat - Méta-analyse}

Épistémologie - ABI/INFORM/Global

\begin{abstract}
L'AUTEUR
PIERRE COSSETTE est professeur titulaire à l'École des sciences de la gestion de l'Université du Québec à Montréal. Après avoir complété un baccalauréat et une maîtrise ès arts en psychologie à l'Université du Québec à Trois-Rivières, il a obtenu une M.B.A. et un Ph. D. en administration de l'Université Laval. L'épistémologie, la méthodologie et les théories de l'organisation sont au cœur de ses intérêts de recherche et d'enseignement. Adresse: École des sciences de la gestion, Université du Québec à Montréal, C.P. 8888, Succ. Centre-Ville, Montréal, Québec, H3C 3P8. Courriel :<cossette.pierre@uqam.ca>.
\end{abstract}

\section{RÉSUMÉ}

Plusieurs chercheurs reconnaissent aujourd'hui l'importance de la dimension cognitive dans le renouvellement des connaissances sur l'entrepreneuriat. Cependant, l'emploi que les chercheurs font du vocabulaire cognitif, en particulier du concept même de cognition, est encore peu documenté. Pourtant, une meilleure connaissance des usages que les chercheurs font de ce concept serait susceptible de mieux faire ressortir les intérêts des chercheurs concernés, de stimuler chez eux la réflexion et la discussion d'ordre théorique associée à l'emploi du concept de cognition en entrepreneuriat ainsi qu'à mieux positionner leurs propres travaux de recherche. La présente étude vise donc à répondre à la question suivante:

1. Cette recherche a fait l'objet d'une communication lors du IX ${ }^{\mathrm{e}}$ Congrès international francophone en entrepreneuriat et PME (CIFEPME) tenu à Louvain-laNeuve (Belgique) en octobre 2008. Je remercie le rédacteur en chef de la revue, Louis Raymond, pour ses commentaires très constructifs ainsi que les trois évaluateurs anonymes pour le travail qu'ils ont accompli. 
Quels usages les chercheurs font-ils du concept de cognition dans leurs travaux sur l'entrepreneuriat? De façon plus précise, l'objectif de cette recherche est de répertorier et caractériser l'ensemble des expressions à l'intérieur desquelles figure le concept de cognition dans la littérature savante sur l'entrepreneuriat. Pour parvenir à faire ainsi le point sur l'utilisation des expressions cognitives par les chercheurs en entrepreneuriat, nous avons consulté la base de données ABI/INFORM Global. En nous appuyant sur les informations obtenues, nous avons pu décrire de manière à la fois globale et précise ce à quoi s'intéressent les chercheurs en entrepreneuriat qui font appel au concept de cognition. Les informations analysées indiquent d'abord que les concepts d'entrepreneuriat et de cognition se trouvent simultanément dans 134 titres ou résumés d'articles publiés depuis 1993 et que leur présence conjointe va en s'accentuant. Sur le plan théorique, ces informations ont permis d'élaborer un cadre de référence spécifique aux chercheurs en entrepreneuriat qui font appel au concept de cognition. Très concrètement, les résultats montrent que les expressions contenant le mot cognition ou un de ses dérivés renvoient souvent à une grande orientation sur le plan théorique ou épistémologique (p. ex., cognitive perspective), parfois à des concepts généraux ( $p$. ex., cognitive factors, cognitive dimension) et, très fréquemment, à des objets particuliers laissant voir ce que la cognition caractérise (p. ex., des systèmes référentiels, des outils, des aptitudes) ou encore comment elle est elle-même caractérisée ( $p$. ex., par son niveau individuel ou collectif, par sa complexité, par sa cohérence). Les limites de cette classification en catégories et sous-catégories sont discutées et différentes voies de recherche sont envisagées.

\section{ABSTRACT}

There is a wide recognition today of the importance of cognition in the renewal of knowledge in entrepreneurship studies. However, our understanding of how scholars make use of the cognitive vocabulary, particularly the concept of cognition, is thus far very limited. Yet, having a better knowledge of such usage would not only bring to light researchers various' interests, but would also stimulate a reflection and initiate a theoretical-oriented discussion about the use of cognition in entrepreneurship research, and help researchers position their own works. Hence, the present study aims to provide an answer to the following research question: How scholars make use of the concept of cognition in their research on entrepreneurship? More particularly, the objective of the research is to identify and characterize the set of expressions containing the concept of cognition in the academic literature on entrepreneurship. To do so, we queried the ABI/INFORM Database. Thus, based on the information obtained, we provided a comprehensive and in-depth description of what attracts the most interest among researchers who use the concept of cognition in the study of entrepreneurship. Interestingly, the analysis of the surveyed articles shows that the concepts of entrepreneurship and cognition are simultaneously present in 134 titles or abstracts of articles that were published after the year 1993, and that their co-presence was ever increasing since then. From a theoretical point of view, the study proposes a reference framework peculiar to entrepreneurship researchers who use the concept of cognition in their research. More specifically, the results show that the expressions containing the word cognition or its derivatives refer to broad theoretical or epistemological orientations (e.g. cognitive perspective). Sometimes, they refer to general concepts (e.g. cognitive factors, cognitive dimension) and, very often, to specific objects that indicate what cognition characterizes (e.g. referential 
systems, tools and aptitudes) or what it is itself characterized by (e.g. individual or group level, complexity and coherence). The limits of the proposed classification in categories and sub-categories are discussed and avenues for future research are presented.

\section{RESUMEN}

Varios investigadores reconocen hoy en día la importancia de la dimensión cognitiva en la renovación del conocimiento sobre el emprendimiento. Sin embargo, el empleo que los investigadores hacen del vocabulario cognitivo y en particular del concepto de cognición ha sido poco documentado. Con todo, el mayor conocimiento en el uso que los investigadores hagan de este concepto, permitiría hacer emerger mejor sus intereses y estimular en ellos la reflexión y el debate de orden teórico asociado al empleo del concepto de cognición en el emprendimiento, así como a mejorar el posicionamiento de sus propios trabajos de investigación. En consecuencia, el presente estudio busca responder a la siguiente pregunta: ¿Qué usos hacen los investigadores del concepto de cognición en sus trabajos sobre el emprendimiento? Más concretamente, el objetivo de esta investigación es inventariar y caracterizar el conjunto de expresiones en las cuales figura el concepto de cognición en de la literatura científica sobre el emprendimiento. Para hacer un balance sobre la utilización de expresiones cognitivas por los investigadores del emprendimiento, se ha consultado la base de datos AB/INFORM Global. Apoyándose en la información obtenida, se puede describir de manera tanto global como precisa, en qué se interesan los investigadores del empredimiento cuando ellos recurren al concepto de cognición. La información analizada indica en primer lugar que los conceptos emprendimiento y cognición se presentan simultáneamente en 134 títulos o resúmenes de artículos publicados desde 1993 en el campo de estudio del emprendimiento y que su presencia conjunta tiende a acentuarse. A nivel teórico, esta información ha permitido elaborar un marco de referencia específico para los investigadores en el emprendimiento que acuden al concepto de la cognición. Muy concretamente, los resultados muestran que las expresiones conteniendo la palabra cognición, o uno de sus derivados, hacen referencia con frecuencia a una gran orientación sobre el plano teórico o epistemológico (ej.: cognitive perspective), a veces se refieren a conceptos generales (ej.: cognitive factors, cognitive dimension) y muy frecuentemente, a objetos particulares que dejan entender lo que caracteriza a la cognición (ej.: los sistemas referenciales, las herramientas, las aptitudes) o incluso muestran cómo ella misma ha sido caracterizada (ej.: por su nivel individual o colectivo, por su complejidad, por su coherencia). Los límites de esta clasificación en categorías y subcategorías se discuten y diferentes vías de investigación son sugeridas.

\section{ZUSAMMENFASSUNG}

Vielerorts wird erkannt, dass die nähere Betrachtung von unternehmerischen Kognitionen den Forschungszweig Entrepreneurship weiterbringen könnte. Die Begrifflichkeiten werden von Forschenden jedoch noch sehr unterschiedlich angewandt. Ziel des Berichts ist demnach, die Gesamtheit der verwendeten Ausdrücke der Literatur zur Thematik zu klassifizieren und zu charakterisieren. Gesamthaft wurden 
134 wissenschaftliche Artikel zur Thematik Entrepreneurship und Kognitionen analysiert, die seit 1993 veröffentlich wurden. Die Literaturübersicht erlaubt eine Gliederung in vier verschiedene Verwendungsarten. Artikel für den erkenntnistheoretischen Gebrauch, allgemeine Konzepte (kognitive Dimensionen, Faktoren etc.), Darstellung von Gegenständlichkeiten dargestellt, die aufzeigen, wie Kognition charakterisiert wird (Referenz-Systeme, Instrumente, Fähigkeiten etc.) oder wie Kognition selber charakterisiert wird (individuelle vs. kollektive Ebene, Komplexität, Kohärenz etc.). Anschliessend werden die Grenzen einer solchen Klassifizierung und verschiedene Forschungsrichtungen diskutiert.

\section{Introduction}

Depuis plus d'une vingtaine d'années, la recherche en entrepreneuriat a connu un développement exceptionnel. Il y a là un champ de recherche qui, dans une large mesure, est aujourd'hui bien formé (Bruyat et Julien, 2001). En témoignent éloquemment l'existence de nombreuses revues consacrées essentiellement ou fortement à l'entrepreneuriat - on pense ici, notamment, à Journal of Business Venturing (JBV), Entrepreneurship Theory and Practice (ETP) et, en français, à Revue internationale PME (RIPME) - ainsi que la quantité impressionnante de manuels de type handbook publiés récemment sur l'entrepreneuriat ou l'une de ses dimensions (internationale, pédagogique, etc.). Il existe aussi des congrès et autres rencontres scientifiques où l'entrepreneuriat occupe une place importante (International Council for Small Business, Congrès international francophone en entrepreneuriat et PME, etc.). On peut noter également que de nombreuses associations reconnues, dont l'Academy of Management et l'Association canadienne des sciences de l'administration, ont une division consacrée à l'entrepreneuriat.

$\mathrm{Au}$ fil des ans, certains chercheurs ont senti le besoin de faire le point sur l'état des connaissances dans ce champ de recherche et de proposer différentes idées ou orientations susceptibles de contribuer à l'enrichissement des connaissances sur l'entrepreneuriat. Parmi ceux-ci, on peut nommer Churchill et Lewis (1986), Wortman (1987), Low et MacMillan (1988), Bygrave (1989a et 1989b), Bygrave et Hofer (1991), Herron, Sapienza et Smith-Cook (1991 et 1992), Hofer et Bygrave (1992), MacMillan et Katz (1992), Bull et Willard (1993), Amit, Glosten et Muller (1993), Filion (1997), Bruyat et Julien (2001), Shane et Venkataraman (2000), Ucbasaran, Westhead et Wright (2001), Low (2001), Davidsson et Wiklund (2001) ainsi que Verstraete et Fayolle (2005). Animés par le désir de mieux comprendre l'entrepreneuriat et l'entrepreneur lui-même, ils se demandent essentiellement comment les entreprises se créent ou se développent, tout en cherchant à mettre en évidence les caractéristiques ou comportements des entrepreneurs. Et, parfois, comme le faisaient Amit, Glosten et Muller (1993), ils déplorent que les théories expliquant pourquoi 
certaines entreprises survivent et d'autres pas, pourquoi certains entrepreneurs réussissent mieux que d'autres et comment ils prennent les décisions auxquelles ils sont confrontés soient encore aussi peu satisfaisantes.

Même si ces grands travaux ont souvent suscité beaucoup d'intérêt, certains trouveront étonnant que, de façon générale, la dimension cognitive n'y occupe, au mieux, qu'une place vraiment marginale, comme si elle n'était pas susceptible de contribuer à une meilleure compréhension du phénomène de l'entrepreneuriat. Pourtant, l'importance de cette dimension cognitive est largement reconnue dans les recherches sur l'organisation, surtout depuis la publication de la 2 é édition de l'ouvrage de Karl Weick, The Social Psychology of Organizing, en 1979. En effet, depuis la parution de cet ouvrage remarquable, volumes et articles dans lesquels elle figure au premier plan ne se comptent plus. Audet et Déry (1996) considèrent même que c'est l'étude de la dimension cognitive qui caractérise la quatrième et plus récente «couche sédimentaire » (p.107) du développement de l'épistémologie des sciences de l'administration, après celles du discours scientiste (début du XX $\mathrm{XX}^{\mathrm{e}}$ siècle), du néopositivisme (fin de la Deuxième Guerre mondiale) et du pluralisme ou diversité des pratiques (à compter des années 1970). Selon eux, depuis les années 1980, il y a un véritable «engouement pour la dimension cognitive des objets de l'administration» (p. 118).

Malgré le peu d'empressement à reconnaître l'importance de cette dimension cognitive dans le renouvellement des connaissances en entrepreneuriat, certains chercheurs ont tout de même clairement choisi d'emprunter cette voie. Par exemple, Busenitz et Lau (1996) proposaient il y a un certain temps déjà un modèle cognitif interculturel de création d'entreprise. De son côté, Baron (1998) faisait état de différents mécanismes cognitifs susceptibles de jouer un rôle important en entrepreneuriat. Quant à Forbes (1999), il insistait sur les processus cognitifs dans la formation de nouvelles entreprises. Citons également Mitchell et al. (2002) pour qui il ne fait pas de doute que les travaux de recherche actuels contribuent au développement d'une théorie de la cognition entrepreneuriale. Sans oublier Baron (2004) qui présente la perspective cognitive comme un outil valable pour répondre aux principaux «pourquoi» de l'entrepreneuriat: Pourquoi certains individus mais pas d'autres décident-ils de devenirs entrepreneurs? Pourquoi certaines personnes mais pas d'autres reconnaissent-elles des opportunités pour de nouveaux produits ou de nouveaux services? Et pourquoi certains entrepreneurs ont-ils plus de succès que d'autres?

Bien sûr, les chercheurs en entrepreneuriat pour qui la dimension cognitive est importante n'ont pas nécessairement recours dans leurs travaux au concept de cognition lui-même. Ainsi, il existe un vocabulaire qu'on pourrait 
qualifier de cognitif au sens large et qui provient en grande partie de disciplines ou domaines comme la psychologie, l'anthropologie et les sciences cognitives, vocabulaire qui comprend évidemment le terme cognition ou ses dérivés (par exemple, cognitif), mais qui comprend aussi d'autres mots dont on reconnaît habituellement la teneur cognitive (par exemple, représentation, intention, signification). Ce langage particulier est extrêmement important parce que c'est à partir de lui que les chercheurs «pensent» et structurent leurs idées en vue d'apporter une contribution théorique dans ce champ de connaissances qu'est l'entrepreneuriat.

Or, l'emploi que les chercheurs font du vocabulaire cognitif est très peu documenté. En d'autres termes, on ne sait pas très bien à quoi s'intéresse exactement l'ensemble des chercheurs pour qui la dimension cognitive est centrale. Pourtant, une connaissance approfondie et «classifiée» du langage de la cognition en entrepreneuriat mettrait en relief les intérêts particuliers des chercheurs concernés. Entre autres, une méta-analyse de ce langage serait susceptible de conduire à l'élaboration d'un cadre de référence spécifique qui pourrait guider les chercheurs en entrepreneuriat dans leurs projets de recherche. L'étude de ce langage montrerait également que certains facteurs, variables, thèmes ou autres objets cognitifs reçoivent de plus en plus d'attention et d'autres, de moins en moins. L'évolution de cet intérêt ou de ce désintérêt pour certains objets de recherche dits cognitifs pourrait suggérer de nouvelles voies de recherche. De façon générale, l'obtention d'une image plus précise du vocabulaire cognitif utilisé dans la recherche en entrepreneuriat serait de nature à stimuler la réflexion et la discussion chez les chercheurs intéressés, de même qu'à orienter leurs propres travaux ou à mieux les positionner par rapport à ceux d'autres chercheurs. Conséquemment, elle serait susceptible de mener à l'émergence de nouvelles idées sur le plan théorique et à une contribution éventuelle au renouvellement des connaissances en entrepreneuriat.

Cependant, répertorier tous les termes constituant ce vocabulaire cognitif, en plus du mot cognition lui-même (et de ses dérivés), serait une tâche absolument colossale et excessivement complexe, même en s'appuyant sur une définition assez précise du concept de cognition. Une première étape, celle qui fera l'objet de la présente recherche, est de se centrer exclusivement sur les expressions contenant le mot cognition lui-même, employé comme nom ou comme adjectif. Cette première étape a un caractère fondamental, étant donné que le mot cognition sert à désigner ou nommer ce concept abstrait (ou non observable) de cognition, ce qui n'est pas le cas d'autres termes parfois considérés comme appartenant au vocabulaire cognitif. Mentionnons aussi que le concept ou la notion de cognition - j'utiliserai indistinctement les deux termes - figure dans des expressions vraisemblablement nombreuses 
et variées (style cognitif, perspective cognitive, cognition entrepreneuriale, cognition symbolique, etc.), ce qui permet d'en faire une analyse approfondie et enrichissante. Simplement à titre illustratif,l'article de Mitchell et al.(2002), qui servait uniquement à introduire un numéro spécial sur le développement d'une théorie de la cognition entrepreneuriale, contient 35 de ces expressions.

Bref, sur le plan théorique, l'analyse de ces expressions repérées dans la littérature sur l'entrepreneuriat peut aider à mieux connaître et comprendre la place qu'occupe l'idée ou le concept de cognition à l'intérieur de cette littérature sur l'entrepreneuriat, même si d'autres mots peuvent aussi être associés à un vocabulaire cognitif. Elle est de nature à mener à l'élaboration d'un cadre de référence particulier et à suggérer de nouvelles pistes de recherche. La présente recherche entend donc faire le point sur l'utilisation des expressions cognitives dans la littérature savante sur l'entrepreneuriat, ce qui devrait permettre d'obtenir une image ou une description à la fois plus globale et plus précise de ce à quoi s'intéressent les chercheurs en entrepreneuriat qui ont recours au concept de cognition.

Cette étude vise à répondre à la question suivante: Quels usages les chercheurs font-ils du concept de cognition dans leurs travaux sur l'entrepreneuriat? De façon plus précise, l'objectif de cette recherche est de répertorier et caractériser l'ensemble des expressions à l'intérieur desquelles figure le concept même de cognition dans la littérature savante sur l'entrepreneuriat, si possible en les regroupant en différentes catégories. En somme, il s'agit de tracer un bilan aussi précis que possible de ce à quoi s'intéressent les chercheurs qui utilisent le concept de cognition - comme substantif ou comme adjectif - dans leurs travaux sur l'entrepreneuriat et du vocabulaire particulier qu'ils emploient pour en rendre compte.

Avant d'aborder les principaux éléments du cadre opératoire de cette étude, apportons quelques précisions sur le concept de cognition, ce qui permettra de situer encore plus clairement la contribution de la présente recherche.

\section{Concept de cognition}

Les concepts riches n'ont pas toujours un sens très précis ou en ont plusieurs, ce qui les rend parfois très attrayants. Comme l'ont montré Astley et Zammuto (1992), de tels concepts peuvent alors être employés de manière créative dans de nombreuses circonstances, ce qui peut être très utile ou enrichissant. Cette constatation convient très bien au concept de cognition.

Même si la notion de cognition est très générale et paraîtra à plusieurs comme un peu ambiguë, y compris à ceux qui en font usage, elle est toujours 
liée à la pensée ou à la connaissance. Le plus souvent, elle désigne une fonction particulière à laquelle sont associés des activités ou des processus se rapportant à l'intelligence humaine. De façon générale, l'étude de la cognition concerne le traitement de l'information ou encore les connaissances qui le permettent ou qui en résultent. Par exemple, Mitchell et al. (2002, p. 97) définissent les cognitions entrepreneuriales comme «des structures de prise de connaissance que les gens emploient pour faire des estimations, pour porter des jugements ou pour prendre des décisions liées à l'évaluation d'opportunité, à la création d'entreprise et à sa croissance ». Cette idée de schème ou de structure cognitive ainsi que celle de mécanismes associés à la formation ou à l'utilisation de ces modèles mentaux (voir, notamment, Baron, 1998 et 2004) sont habituellement au cœur des travaux de la plupart des chercheurs en entrepreneuriat s'intéressant à la cognition, même s'ils définissent rarement ce concept de façon très claire.

Dans les études sur l'organisation, Meindl, Stubbart et Porac (1994) utilisaient les catégories products, processes et aids pour classifier les travaux sur la cognition. De leur côté, Schneider et Angelmar (1993) adoptaient un cadre de référence constitué de trois composantes: des structures cognitives, des processus cognitifs et des styles cognitifs. Quant à Cossette (2004), il proposait de regrouper les objets cognitifs en quatre grandes catégories: les produits, les processus, les prédispositions et les procédés. Ces modèles de classification aident certainement à s'y retrouver un peu dans cette imposante littérature dite scientifique, mais ils ne nous renseignent pas beaucoup sur l'usage que les chercheurs eux-mêmes font du concept de cognition.

Comme l'ont bien noté Meindl, Stubbart et Porac (1994), il n'y a pas de nomenclature de termes cognitifs, ni même de consensus sur ce qui est cognitif et sur ce qui ne l'est pas. Il semble exister un vocabulaire étendu que certains associent à la cognition (intentions, croyances, langage, interprétation, représentation, apprentissage, schèmes, etc.), mais le mot cognition lui-même (ou ses dérivés) n'en fait souvent pas partie. Par exemple, dans une impressionante synthèse, Walsh (1995) dressait un tableau censé refléter le langage employé dans les écrits sur la «cognition managériale et organisationnelle» (p.284). Or, parmi les expressions tirées de 77 références différentes figurant dans ce tableau, seulement les six suivantes contiennent le mot cognition ou cognitive: cognitive maps, cognitive biases, cognitive frameworks, cognitive trails, collective cognitions et cognitive inertia.

Par ailleurs, dans une étude visant à mettre en évidence les caractéristiques des travaux de recherche en sciences de l'administration dans lesquels le terme cognition ou ses dérivés occupent une place importante, Ouellet et Cossette (1999) avaient repéré dans les résumés des articles un 
total impressionnant de 133 expressions de cette nature, dont 86 qui n'avaient été utilisées qu'une seule fois. Les auteurs s'inquiétaient du développement apparemment anarchique du langage associé à la cognition, ce qui, selon eux, pouvait témoigner d'une certaine confusion sur le plan intellectuel. Ils signalaient aussi que plusieurs des termes employés (perspective, approche, etc.) n'avaient pas toujours une signification très claire, même s'ils n'ont pas analysé en profondeur le sens attribué à ces expressions.

Qu'en est-il exactement dans la littérature savante sur l'entrepreneuriat? C'est ce qui sera examiné dans la présente recherche. Étant donné qu'il ne semble exister aucun cadre de référence de nature linguistique qui pourrait guider l'analyse des usages du concept même de cognition dans les travaux de recherche sur l'entrepreneuriat, nous allons suivre une logique inductive, c'est-à-dire partir de chaque cas particulier (donc, de chaque usage du concept de cognition), avant de généraliser de façon plus ou moins étendue, notamment par la création de catégories et sous-catégories. En d'autres termes, tout effort de classification ou de théorisation s'appuiera sur des données singulières tirées du repérage de toutes les expressions «cognitives». Cette approche est particulièrement appropriée dans le cas d'une méta-analyse de type exploratoire, c'est-à-dire ne s'appuyant pas sur un ensemble de catégories prédéterminées (pour un excellent exemple d'une telle recherche inductive, voir Partington et Jenkins, 2007).

\section{Cadre méthodologique}

Pour atteindre l'objectif de la présente étude, il fallait d'abord répertorier les travaux de recherche sur l'entrepreneuriat à l'intérieur desquels le concept de cognition occupait une place importante. Pour y arriver, la base de données ABI/INFORM Global (diffusée par ProQuest) fut consultée. Cette base de données, qui existe depuis 1971 (même si l'on peut y retracer des textes antérieurs à cette année), contient des informations relatives aux articles publiés dans plus de 1800 périodiques, presque tous anglophones. Ces revues, autant scientifiques que professionnelles, couvrent les principaux domaines de la gestion et des affaires, y compris la comptabilité, la finance, l'économique, la stratégie, le marketing, les ressources humaines, les systèmes d'information et le comportement organisationnel. Outre la référence complète de chaque article qu'elle permet de repérer, ABI/INFORM Global fournit également le résumé (abstract) et quelques autres informations.

Cette base de données fut questionnée (la dernière fois le 18 février 2008, mais sans jamais fixer de date de départ) à partir de l'énoncé suivant: [entrepreneur* and cogniti*]. La troncation permettait de repérer les mots ayant 
la même racine (cognition, cognitive, entrepreneurship, entrepreneurial, etc.), donc renvoyant au même concept. Seuls les textes publiés jusqu'en 2007 dans des revues avec arbitrage (peer reviewed) furent pris en considération.

L'énoncé de recherche visait à repérer toutes les notices bibliographiques d'ABI/INFORM Global contenant les deux termes (ou leurs dérivés) dans le résumé ou dans le titre uniquement, ce qui laissait croire que la cognition et l'entrepreneuriat occupaient une place vraiment importante dans cette recherche. Bien sûr, il n'est pas impossible que ces deux concepts aient réellement fait l'objet d'une attention particulière dans un article sans figurer dans le résumé ou le titre, ou encore qu'ils n'aient pas été au cœur d'une recherche tout en figurant dans le résumé ou le titre, mais c'est très improbable; si tel était le cas, ce serait le résumé (et le titre ?) qu'il faudrait mettre en cause.

\section{Résultats}

Le tableau 1 montre comment a évolué au cours des ans le nombre d'articles dans lesquels le titre ou le résumé contient les concepts d'entrepreneuriat et de cognition. On constate d'abord que l'occurrence simultanée de ces deux concepts n'a débuté qu'en 1993 et qu'elle a connu une croissance tout simplement fulgurante. Parmi les 134 articles répertoriés, plus de la moitié (72, soit 53,7 \%) ont été publiés depuis 2004, c'est-à-dire au cours des quatre dernières années. De toute évidence, la cognition occupe une place grandissante et fondamentale dans la littérature savante sur l'entrepreneuriat.

TABLEAU 1

Nombre d'articles dans lesquels le titre ou le résumé contient les concepts d'entrepreneuriat et de cognition

\begin{tabular}{cccccc}
\hline 1993 & 2 & 1998 & 5 & 2003 & 8 \\
\hline 1994 & 1 & 1999 & 7 & 2004 & 19 \\
\hline 1995 & 5 & 2000 & 10 & 2005 & 8 \\
\hline 1996 & 4 & 2001 & 6 & 2006 & 20 \\
\hline 1997 & 2 & 2002 & 12 & 2007 & 25 \\
\hline
\end{tabular}

Comment expliquer une telle augmentation? Il est probable que l'importance croissante accordée à la dimension cognitive dans l'étude des organisations en général se soit reflétée dans la recherche en entrepreneuriat. Mais il faut aussi reconnaître qu'il y a de plus en plus de recherches sur l'objet 
de l'entrepreneuriat lui-même, ce qui augmente de facto la probabilité d'un plus grand nombre de recherches dans lesquelles on fait appel au concept de cognition.

Combien d'expressions cognitives différentes ces 134 titres et résumés contiennent-ils? Difficile de répondre précisément à cette question, étant donné que certaines d'entre elles sont très semblables ou quasi identiques (p. ex., entrepreneurial cognition research, entrepreneurial cognition inquiry, entrepreneurial cognition research stream). Mais on peut affirmer sans trop risquer de se tromper qu'il y en aurait une centaine, ce qui signifie que plusieurs d'entre elles ne sont employées qu'à une seule occasion, même si certains résumés en contiennent deux, parfois davantage.

Cependant, le plus grand défi qui se pose ici n'est pas d'obtenir un chiffre précis révélant le nombre d'expressions cognitives vraiment différentes. Il consiste plutôt à les regrouper de façon intelligible, c'est-à-dire à les classifier de manière à leur donner un sens dans leur ensemble. Cette tâche est d'autant plus complexe que les expressions dans lesquelles figure le concept de cognition sont d'une variété impressionnante.

L'examen attentif de ces expressions cognitives suggère de les regrouper essentiellement en trois catégories. Cette proposition d'un cadre de référence spécifique aux chercheurs en entrepreneuriat qui utilisent le concept de cognition met en relief le caractère très disparate des usages qu'ils font de ce concept de cognition. Ainsi, il peut être associé à une grande orientation, à des concepts généraux ou encore à des objets particuliers, ce que nous allons maintenant regarder de plus près.

\subsection{Cognition : une grande orientation}

Plusieurs expressions répertoriées évoquent une «façon» d'aborder l'étude de l'entrepreneuriat, une grande orientation. On peut subdiviser cette catégorie en trois sous-ensembles.

D'abord, le concept de cognition est parfois employé pour désigner un champ d'appui, c'est-à-dire un champ de connaissances ou encore une discipline permettant de positionner de manière très générale une recherche sur l'entrepreneuriat. Les expressions cognitive science (ou cognitive sciencebased investigation), cognitive psychology (ou cognitive developmental psychology) ou cognitive anthropology rendent bien compte de cette orientation.

Ensuite, il y a certaines expressions qui témoignent de l'existence de une ou plusieurs orientations précises (mais pas nécessairement bien précisées) sur le plan théorique ou épistémologique. Ces expressions, dont la liste est 
présentée plus bas, sont associées à ce que l'on considère habituellement comme le «cadre» théorique ou épistémologique de la recherche. Notons que la plupart d'entre elles sont employées tantôt au singulier, comme s'il n'y avait qu'une seule perspective, approche, théorie, etc., qui soit «cognitiviste», tantôt au pluriel, comme si plusieurs pouvaient l'être, ce qui peut créer une certaine confusion:

- Cognitive perspective (ou cognitive-affective perspective);

- Cognitive approach (ou cognitive diversity approach);

- Cognitive paradigm;

- Cognitive theory (ou psycho-cognitive theory, social-cognitive theory, social cognition literature, transactions cognition theory);

- Cognitive school (ou cultural cognitive school);

- Cognitive framework (ou social cognitive framework);

- Cognitive/agency-oriented view;

- Cognitive model (ou cross-cultural cognitive model).

Finalement, d'autres expressions renvoient, le plus souvent très directement, à l'étude de la cognition entrepreneuriale:entrepreneurial cognition (que ces mots soient suivis ou non du terme research, research stream, inquiry, domain ou canon), theories of entrepreneurial cognitions ou, dans un sens très rapproché, human cognition et managerial cognition. Ces expressions montrent simplement que la cognition constitue un objet d'étude général - un champ de connaissances, diraient certains, dont Hindle (2004) - à l'intérieur du domaine de l'entrepreneuriat. En ce sens, elles reflètent une orientation donnée à certaines études sur l'entrepreneuriat.

\subsection{Cognition : des concepts généraux}

Les concepts cognitifs généraux occupent une place importante dans les expressions repérées dans les titres et résumés d'articles sur l'entrepreneuriat. Au premier chef se trouve le concept de cognition lui-même, un concept très général comme nous le mentionnions précédemment et dont le sens n'est pas toujours évident ou bien précisé. Mais il y a plusieurs autres expressions construites à partir de l'adjectif cognitive et renvoyant à des concepts généraux: factors, dimension, aspects, phenomena, concepts, variables, constructs, criteria, world, issues, knowledge, source, predictors, repertories, underpinnings, bases, etc. 
L'expression cognitive process (ou cognitive reasoning process), qui désigne également une notion très générale, se situe dans une classe à part. Repérée à huit reprises - l'occurrence la plus fréquente dans la présente recherche -, elle semble essentiellement associée aux mécanismes ou activités mettant en évidence le fonctionnement de l'esprit. Dans les articles sur l'entrepreneuriat, l'expression cognitive process se rapporte souvent à la reconnaissance ou à l'identification d'opportunités ou encore à l'étude des heuristiques ou règles informelles (ou intuitives) suivies pour prendre une décision ou résoudre un problème. Bien que l'expression ne figure dans aucun titre ou résumé d'articles sur l'entrepreneuriat, les heuristiques cognitives ont été abondamment étudiées dans ce champ de connaissances. Étroitement rattachées aux biais cognitifs, elles constitueraient des stratégies de simplification susceptibles de se manifester, notamment, lorsque l'information est surabondante, en contexte d'incertitude et quand le temps est un facteur important (Busenitz et Lau, 1996; Baron, 1998). L'expression cognitive mechanism est également employée dans le résumé d'un article pour rendre compte des heuristiques cognitives.

\subsection{Cognition: des objets particuliers}

Nous entrons ici dans une catégorie qui requiert un travail de classification plus élaboré. Ainsi, dans la littérature sur l'entrepreneuriat (et ailleurs, pourrait-on ajouter), on peut d'abord constater que le concept de cognition est employé dans au moins deux types d'expressions pour désigner des objets particuliers. Dans le premier, de loin le plus fréquent, l'usage du concept de cognition vise à caractériser un autre objet d'intérêt (p. ex., cognitive style); dans un tel cas et en rappelant que la présente étude ne porte que sur les écrits anglophones, c'est généralement l'adjectif cognitive qui est utilisé. Dans le second type, c'est la cognition elle-même qui est caractérisée (p. ex., collective cognition); le plus souvent, c'est le substantif cognition qui est alors utilisé dans de telles expressions. Dans cette section, nous allons traiter distinctement les deux.

\subsubsection{Qu'est-ce que la cognition caractérise?}

Qu'est-ce qui est «cognitif» exactement dans la littérature savante sur l'entrepreneuriat? Dit autrement, quels sont les objets qualifiés de cognitive dans la recherche sur l'entrepreneuriat, en excluant les grandes orientations abordées dans la section précédente? 
En se concentrant sur ceux qui ont reçu le plus d'attention, on peut classifier les principaux objets dits cognitifs en six groupes: des systèmes référentiels, des outils, des aptitudes et habiletés, des styles, des biais et des changements. Voyons cela de plus près.

\subsubsection{Systèmes référentiels}

Lorsque les chercheurs en entrepreneuriat emploient le terme cognitive, très souvent, c'est pour désigner un «schème» ou une «structure mentale » guidant l'entrepreneur dans son appréhension de la réalité, même si les chercheurs ne recourent à peu près jamais à ces deux termes dans le titre ou dans le résumé de leur texte. Dans ce sens, les termes qui accompagnent le plus fréquemment le mot cognitive sont framework (un terme qui est aussi employé pour désigner une école de pensée, comme on l'a constaté précédemment), representation, mindset, frame, structure, institutional structure, institution et infrastructure. Le terme cognition lui-même est parfois employé pour désigner une structure cognitive, comme on l'a vu, par exemple, dans la définition des cognitions entrepreneuriales proposée par Mitchell et al. (2002, p. 97). Les chercheurs se servent habituellement de tous ces termes comme s'ils étaient synonymes. L'expression cognitive script, repérée dans le résumé de deux articles publiés en 2000 et 2007, représente un type particulier de schème mettant en évidence une séquence typique d'événements, d'actions ou d'idées dans une situation donnée.

\subsubsection{Outils}

Plusieurs chercheurs font également appel au concept de cognition pour qualifier des «outils» susceptibles d'être utiles en contexte de recherche ou d'intervention. Dans l'usage qui en est fait, ce caractère instrumental se manifeste surtout sous son aspect technique (cognitive techniques, cognitive methods, cognitive tools, entrepreneurial cognition's toolbox), mais il existe également sur le plan conceptuel (cognitive machinery, cognitive strategy). Chacune de ces expressions n'a été repérée qu'à une seule occasion. Mais le concept de cognitive map, employé pour désigner un de ces outils, a été vu dans le résumé de trois articles, parus en 1999, 2000 et 2009.

\subsubsection{Aptitudes et habiletés}

Dans les titres ou résumés des articles sur l'entrepreneuriat, on fait état de cognitive skills (une seule occurrence) et, surtout, de cognitive abilities (six occurrences, toutes depuis 2001), qui seraient des aptitudes ou des capacités à apprendre, comme si c'étaient elles qui rendaient possible l'acquisition 
d'habiletés. Mentionnons également que la veille entrepreneuriale (entrepreneurial alertness), un concept lié dans un texte à un ensemble d'habiletés particulières, a été décrite comme le cognitive engine dans le processus d'identification d'opportunités.

\subsubsection{Styles}

Le concept de style cognitif figure dans six titres ou résumés d'articles portant sur l'entrepreneuriat. Dans tous les cas, les auteurs et coauteurs de ces six textes sont différents, ce qui laisse penser que ce concept suscite un très grand intérêt chez ceux qui étudient l'entrepreneuriat. Signalons qu'un seul de ces textes a été publié avant 2004, ce qui montre que cet engouement pour l'étude des styles cognitifs des entrepreneurs est très actuel. L'expression cognitive system a également été utilisée pour montrer la façon particulière dont l'entrepreneur traite l'information sur le plan cognitif.

\subsubsection{Biais}

Les biais cognitifs désignent généralement des tendances reflétant chez l'individu des idées préconçues ou des erreurs dans la façon de traiter l'information, le plus souvent en lien avec le processus décisionnel. Par exemple, un biais possible chez l'entrepreneur serait de surestimer la probabilité de succès et de sous-estimer la probabilité d'échec. Dans la présente recherche, l'expression cognitive bias a été trouvée dans cinq titres ou résumés. Elle est directement liée aux cognitive errors, une expression repérée en trois occasions (aucune après l'an 2000).

\subsubsection{Changements}

Si l'on se fie au titre ou au résumé des articles publiés sur l'entrepreneuriat, le changement cognitif reçoit passablement d'attention. Les principales expressions témoignant de cet intérêt pour la transformation de la pensée sont les suivantes: cognitive change, cognitive transformation, cognitive shift, cognitive development et cognitive modulation. Chacune d'elles n'a été employée qu'une seule fois.

D'autres objets sont également «étiquetés » comme cognitifs dans les titres ou résumés d'articles sur l'entrepreneuriat, selon l'usage qu'en font certains chercheurs du moins. On les retrouve dans les expressions suivantes:

- Cognitive effects, cognitive influence, cognitive benefits;

- Cognitive explanation, cognitive understanding; 
- Cognitive legitimacy, cognitive legitimation;

- Cognitive involvement, cognitively committed relationship;

- Cognitive mediation;

- Cognitive experience;

- Cognitive differences;

- Cognitive leadership;

- Cognitive conflict;

- Socio-cognitive integrity;

- Cognitive proximity;

- Self-esteem cognition;

- Cognitive context.

\subsubsection{Comment la cognition est-elle caractérisée?}

Sans tenir compte encore une fois des occurrences liées aux grandes orientations dont il fut question dans la sous-section 3.1, comment la cognition elle-même est-elle caractérisée dans les travaux de recherche sur l'entrepreneuriat? Le plus souvent, on obtient une réponse à cette question en examinant les adjectifs employés pour qualifier le substantif cognition. Cependant, dans la langue anglaise du moins, une caractéristique de la cognition peut parfois être tirée d'une expression dans laquelle l'adjectif cognitive est joint à un substantif qui n'est pas cognition, comme dans cognitive coherence, la cognition étant alors caractérisée par sa cohérence plus ou moins grande.

Pour étudier ces caractéristiques de la cognition, considérons qu'un adjectif est dit classifiant lorsqu'il «attribue une catégorie à l'être ou à l'objet désigné par le nom qu'il accompagne » (p. ex., des cours obligatoires, un animal invertébré) ou qualifiant lorsqu'il «exprime une qualité de l'être ou de l'objet désigné par le nom qu'il accompagne» (p. ex., une analyse minutieuse, des avis discutables), conformément à ce qu'en dit le Multidictionnaire de la langue française (de Villers, 2009, p. 36). Les deux ne sont pas toujours faciles à distinguer, mais pour nous aider et en nous rapportant de nouveau à la même source, reconnaissons qu'un adjectif classifiant ne peut jamais être accompagné d'un adverbe modérateur exprimant divers degrés, contrairement à un adjectif qualifiant. Par exemple, un cours ne peut pas être «très» obligatoire. 


\subsubsection{Adjectifs classifiants}

En plus des adjectifs classifiants qu'on retrouve dans les expressions désignant une grande orientation (entrepreneurial cognition, human cognition, managerial cognition), les principaux adjectifs classifiants repérés dans la littérature sur l'entrepreneuriat renvoient aux différents niveaux d'analyse de l'entrepreneuriat: individual cognition, collective cognition et team cognition (ou entrepreneurial team's social cognition) sont des expressions qui rendent bien compte de cet usage. Mais il y a au moins deux autres adjectifs qui peuvent aussi être considérés comme classifiants et qui sont utilisés pour caractériser la cognition: cross-cultural cognitions et enacted cognition.

\subsubsection{Adjectifs qualifiants}

La cognition semble avoir plusieurs «qualités» ou manières d'être. Les expressions qui en rendent compte sont les suivantes: creative cognition, symbolic cognition, semantic cognition, conflicting cognitions, mais aussi cognitive coherence, cognitive complexity, cognitive comprehensiveness et cognitive embeddedness. Dans ces dernières expressions construites à partir de l'adjectif cognitive, ce n'est pas vraiment la cohérence, la complexité, etc., qui sont plus ou moins cognitives, mais bien la cognition qui se caractérise par sa plus ou moins grande cohérence, complexité, etc.

\section{Discussion et conclusion}

L'objectif de la présente étude était de déterminer les usages que les chercheurs font de la notion de cognition dans leurs travaux sur l'entrepreneuriat en répertoriant et caractérisant toutes les expressions contenant le mot cognition ou un de ses dérivés dans le titre ou le résumé de ces articles. Les résultats ont montré que ces expressions, dont le nombre a connu une croissance exceptionnelle au fil des ans, sont d'une diversité remarquable. Ils ont donné lieu à la proposition d'un nouveau cadre de référence spécifique aux chercheurs en entrepreneuriat qui ont recours au concept de cognition pour réaliser leurs travaux de recherche. Ce modèle révèle que les expressions cognitives utilisées renvoient souvent à une grande orientation (p. ex., cognitive perspective), mais aussi à des concepts très généraux comme cognitive factors ou cognitive dimension. Cependant, plus fréquemment, ces expressions désignent des objets particuliers et laissent voir ce que la cognition «caractérise» exactement (des systèmes référentiels, des outils, des aptitudes, etc.) ou encore comment elle est elle-même caractérisée (par son 
niveau individuel ou collectif, par son aspect créatif, par sa cohérence, etc.). Ces objets particuliers paraissent s'inscrire aussi bien dans le courant de la cognition sociale où l'accent est mis sur la façon dont les gens donnent un sens à leur réalité (on pense ici surtout aux systèmes référentiels), que dans celui de la théorie de la décision (behavioral decision theory), où l'accent est plutôt placé sur le processus décisionnel et ses imperfections (on pense ici principalement aux biais cognitifs); selon Tenbrunsel et al. (1996), c'est dans ces deux grands champs de recherche que prennent place les études sur la dimension cognitive dans les organisations.

Bref, cette recherche a mis en relief ce à quoi s'intéressent les chercheurs en entrepreneuriat qui font appel au concept de cognition. Elle a conduit à une classification à la fois globale et précise des expressions cognitives auxquelles ils font appel. Ce faisant, les résultats obtenus pourront aider les chercheurs dans l'orientation qu'ils veulent donner à leur recherche ou dans le choix d'un objet particulier. Ils pourront également leur permettre de mieux positionner ou situer leurs propres travaux par rapport à ceux déjà réalisés par d'autres.

Comme on a pu le constater, les expressions dites cognitives sont nombreuses et ne sont souvent employées qu'une seule fois. Ce qui amène à se demander s'il n'y a pas une certaine inflation dans le vocabulaire utilisé. Par exemple, de nombreuses expressions repérées sont quasi pléonastiques (human cognition, cognitive perception, cognitive knowledge, cognitive understanding, etc.), ce qui semble ajouter plus de confusion que de précision.

Peut-être plus important encore, il faudrait examiner le sens attribué par différents auteurs à ces expressions contenant le concept de cognition. Par exemple, les expressions cognitive perspective, cognitive approach et même cognitive school, cognitive model, cognitive paradigm, cognitive theory et cognitive framework semblent fréquemment employées indistinctement. Est-ce possible, souhaitable ou approprié que des expressions différentes désignent la même réalité ? La clarté et la précision ne sont-ils pas des défis importants qui concernent tout chercheur? Starbuck (1999) affirme sans hésiter que l'usage de synonymes comme substituts d'expressions ou de mots importants dans une recherche est à éviter, parce qu'ils peuvent créer de la confusion. Weick (1995) reconnaît aussi que «différents mots signifient différentes choses» (p. 293), tout en soutenant par ailleurs que l'emploi de synonymes peut aider à donner à une idée une portée plus générale parce que l'idée devient alors moins précise. Le moins auquel on puisse s'attendre est que le chercheur indique clairement qu'il utilise indistinctement ou non les deux termes ou expressions particulières. 
Cette surabondance de mots ou d'expressions contenant le concept de cognition ou ses dérivés dans la littérature sur l'entrepreneuriat reflète probablement un état de grand enthousiasme pour la dimension cognitive, comme l'avait déjà noté Walsh (1995) dans son travail portant sur l'ensemble des études en gestion. Mais, en même temps, elle fait ressortir la nécessité que les chercheurs concernés précisent davantage le sens des termes qu'ils utilisent. Ce qui devrait faciliter la communication et le développement théorique de la conversation savante sur l'entrepreneuriat. Bref, comme on s'attend généralement de tout chercheur qu'il adopte une attitude «obsessive » et qu'il accorde beaucoup d'attention aux détails et au choix des mots (Bem, 2003), le champ de connaissances en entrepreneuriat ne pourra que s'enrichir d'une plus grande précision dans le vocabulaire des chercheurs qui font appel au concept de cognition.

La classification élaborée ici, principalement la grande catégorie et les sous-catégories des objets particuliers de la cognition, ne permet pas de déterminer si l'approche adoptée par les chercheurs pour étudier ces objets cognitifs était de type inductif ou déductif. En d'autres termes, pour étudier les systèmes référentiels, les aptitudes et habiletés, les styles cognitifs, etc., les chercheurs en entrepreneuriat ont-ils tendance à privilégier une approche «du bas vers le haut» (bottom-up), c'est-à-dire inductive ou fondée sur les données (data-driven), ou une approche «du haut vers le bas» (top-down), c'est-à-dire déductive ou guidée par la théorie existante (theory-driven), une distinction traitée de manière stimulante par Walsh (1995, p. 281-282) et par Langley (1999, p. 707-708)? Une meilleure connaissance de l'orientation dominante prise par les chercheurs en entrepreneuriat aiderait à mieux connaître et comprendre l'état actuel du développement théorique lié à ces objets cognitifs particuliers, donc à bien voir ce qui n'a pas été fait et ce qu'il serait peut-être intéressant de faire. Par exemple, si l'on constatait que la recherche sur les systèmes référentiels des entrepreneurs avait surtout porté jusqu'à maintenant sur l'impact de ces structures cognitives sur le traitement de l'information (donc approche «du haut vers le bas»), certains pourraient décider d'examiner de plus près comment elles se constituent et se transforment (donc, approche «du bas vers le haut»), un aspect qui aurait été négligé.

Cette recherche n'a porté que sur le concept même de cognition, c'est-àdire sur le mot lui-même et sur ses dérivés. Cette première étape est importante, mais elle ne doit pas faire oublier que plusieurs termes autour de ce construit fondamental peuvent également être considérés de nature cognitive (valeurs, croyances, représentations, perceptions, intentions, visions, etc.), même s'ils ne figurent habituellement pas à l'intérieur d'expressions 
contenant le mot cognition ou cognitive. Bien sûr, comme l'ont bien noté Meindl, Stubbart et Porac (1994), il n'y a pas de nomenclature de termes cognitifs, ni même de consensus sur ce qui est cognitif et sur ce qui ne l'est pas. Malgré tout, pour obtenir une connaissance plus fidèle et approfondie de la place qu'occupe la cognition dans ce champ de connaissances qu'est l'entrepreneuriat, des recherches ultérieures devraient se pencher sur tout le vocabulaire dit «cognitif», que le mot cognition (ou des dérivés) fasse partie ou non des termes répertoriés. Ce qui permettra d'enrichir ou de préciser encore davantage le cadre de référence proposé dans la présente recherche.

Les expressions cognitives répertoriées ici sont celles qui figurent dans le titre ou le résumé des articles, mais elles ne constituent qu'une partie de toutes celles qu'on retrouve à l'intérieur des textes évoqués sur l'entrepreneuriat. Il est possible que ces autres expressions non répertoriées dans la présente recherche ne soient pas sans intérêt, mais on peut penser qu'elles n'étaient pas considérées comme fondamentales par leurs auteurs, pas suffisamment du moins pour qu'ils décident de les faire figurer dans le titre ou le résumé de leur article. Il serait tout de même enrichissant de les recenser si l'on souhaite dresser un portrait plus précis des expressions cognitives dans la littérature savante sur l'entrepreneuriat et améliorer le cadre de référence proposé.

La présente étude s'est limitée aux expressions de langue anglaise telles qu'elles ont été répertoriées dans la base de données ABI/INFORM Global. Bien que cette base de données fasse autorité, il faut reconnaître l'existence d'autres bases de données et de textes publiés dans d'autres langues. Entre autres, il y a dans la francophonie une communauté de chercheurs très active dans le champ de l'entrepreneuriat, certains faisant appel au concept de cognition dans leurs travaux. Il serait peut-être intéressant de recenser les expressions qu'ils utilisent et de les comparer à celles employées dans des textes en anglais. Très concrètement, en quoi se ressemblent-elles et en quoi sont-elles différentes? Les deux communautés linguistiques sont-elles vraiment distinctes à cet égard? Mais il ne faudrait surtout pas oublier que la traduction d'une expression d'une langue à une autre ne peut pas toujours rendre avec exactitude le sens qu'elle avait au départ, comme si l'expression originale et sa traduction n'avaient pas la même résonance sémantique... Par ailleurs, en consultant à partir de l'énoncé [entrepreneur* et cogniti*] la base de données REPÈRE, qui inclut 495000 références d'articles de périodiques de langue française (1980-), on ne trouve que quatre références dont le titre ou le résumé contiennent ces deux concepts. La plus récente date de l'an 2000 et trois d'entre elles proviennent de la RIPME. 
Baron (2004) se demandait pourquoi certains individus décident de devenir entrepreneurs, pourquoi certains sont plus habiles que d'autres à reconnaître des opportunités et pourquoi certains ont plus de succès. Les résultats de cette recherche ne visaient pas à répondre à de telles questions invitant à comparer des individus. Mais les éléments du cadre de référence proposé ici suggèrent certains points de départ pour étudier ces questions. Les caractéristiques des systèmes référentiels des individus (notamment celles des scénarios ou scripts suivis en contexte entrepreneurial), les aptitudes et habiletés requises de même que les styles cognitifs plus ou moins appropriés pour différentes tâches entrepreneuriales constituent des objets de recherche susceptibles d'apporter des réponses aux questions de Baron. À l'étude de ces schèmes, compétences et styles cognitifs, on pourrait ajouter qu'une meilleure connaissance du rôle de la mémoire et de l'apprentissage d'une expérience entrepreneuriale à l'autre pourrait aider à comprendre pourquoi certains entrepreneurs et pas d'autres deviennent clairement meilleurs au fil de leurs expériences. Ces objets cognitifs, dont l'importance n'est pas évidente dans la classification proposée ici, pourraient bien avoir été négligés dans la recherche en entrepreneuriat.

Finalement, l'évolution des expressions utilisées au fil des ans n'a pas fait l'objet d'une analyse dans la présente recherche. Pourtant, on réalise rapidement que certaines expressions sont récentes (p. ex., cognitive institutional structures, creative cognition, cognitive involvement), que d'autres paraissent de plus en plus en plus employées (p. ex., cognitive style, cognitive abilities, cognitive bias) et que quelques-unes semblent l'être un peu moins (p. ex., cognitive map). Cette constatation indique-t-elle des directions prises par les chercheurs sur l'entrepreneuriat qui ont recours à la notion de cognition? Entre autres, l'analyse réalisée ici montre que le concept de «style cognitif » a fait l'objet de nombreuses recherches au cours des dernières années et, de manière un peu étonnante, par des chercheurs tous différents. Ce qui laisse penser que l'intérêt pour cet objet cognitif par les chercheurs en entrepreneuriat ne semble pas prêt de s'éteindre (comme point de départ, je suggère au lecteur intéressé d'examiner le texte de Sadler-Smith, 2004). On peut s'attendre à ce que de nouvelles études explorent encore davantage les styles cognitifs des entrepreneurs, la pertinence de chacun d'eux selon les circonstances et leur lien avec d'autres caractéristiques des entrepreneurs (attitude à l'égard du risque, tolérance à l'ambiguïté, etc.). Ces styles cognitifs pourraient également être mis en relation avec différentes variables liées à la reconnaissance ou à la construction d'opportunités d'affaires (p. ex., prédisposition à s'engager dans ce processus, succès obtenu) ou encore avec les heuristiques suivies en situation de prise de décision ou de résolution de problème. 
Il semble que le renouvellement des connaissances sur l'entrepreneuriat passe de plus en plus par l'adoption du concept de cognition. La présente étude constitue un bilan de ce à quoi les chercheurs s'intéressent lorsqu'ils font appel à ce concept dans leurs travaux sur l'entrepreneuriat. Il faudra l'enrichir encore et le mettre à jour périodiquement, ce qui permettra d'améliorer le cadre de référence proposé ici.

\section{Bibliographie}

Amit, R., L. Glosten et E. Muller (1993), «Challenges to theory development in entrepreneurship research », Journal of Management Studies, vol. 30, $\mathrm{n}^{\circ}$ 5, p. 815-834.

Astley, W.G. et R.F. ZAMmUTO (1992), «Organization science, managers, and language », Organization Science, vol. 3, n ${ }^{\circ}$ 4, p. 443-460.

AUDET, M. et R. DÉRY (1996), «La science réfléchie. Quelques empreintes de l'épistémologie des sciences de l'administration », Anthropologie et Sociétés, vol. 20, $\mathrm{n}^{\mathrm{o}} 1$, p. 103-123.

BARON, R.A. (1998), «Cognitive mechanisms in entrepreneurship: why and when entrepreneurs think differently than other people», Journal of Business Venturing, vol. 13, $\mathrm{n}^{\circ}$ 4, p. 275-294.

BARON, R.A. (2004), «The cognitive perspective: a valuable tool for answering entrepreneurship' basic “why" questions», Journal of Business Venturing, vol. 19, $\mathrm{n}^{\mathrm{o}} 2$, p. 221-239.

BEM, D.J. (2003), «Writing the empirical journal article», <http://dbem.ws/ WritingArticle.2.pdf>.

BRUYAT, C. et P.-A. JULIEN (2001), «Defining the field of research in entrepreneurship », Journal of Business Venturing, vol. 16, nº 2, p. 165-180.

BULL, I. et G.E. WILLARD (1993), «Towards a theory of entrepreneurship», Journal of Business Venturing, vol. 8, nº 3, p. 183-195.

BusenitZ, L.W. et C.-M. LAU (1996), «A cross-cultural cognitive model of new venture creation», Entrepreneurship Theory and Practice, vol. 20, n 4, p. 25-39.

BYGRAVE, W.D. (1989a), «The entrepreneurship paradigm (I): a philosophical look at its research methodologies», Entrepreneurship Theory and Practice, vol. 14, $\mathrm{n}^{\circ} 1$, p. 7-26.

BYGRAVE, W.D. (1989b), «The entrepreneurship paradigm (II): chaos and catastrophes among quantum jumps?», Entrepreneurship Theory and Practice, vol. 14, $\mathrm{n}^{\circ} 2$, p. 7-30.

BYgRAVE, W.D. et C.W. Hofer (1991), «Theorizing about entrepreneurship», Entrepreneurship Theory and Practice, vol. 16, $\mathrm{n}^{\circ}$ 2, p. 13-22. 
Churchill, N.C. et V.L. LEWIS (1986), «Entrepreneurship research: directions and methods », dans D.L. Sexton et R.W. Smilor (dir.), The Art and Science of Entrepreneurship, Cambridge, Ballinger, p. 333-365.

CossetTe, P. (2004), L'organisation. Une perspective cognitiviste, Québec, Les Presses de l'Université Laval, coll. «Sciences de l'administration».

DAVIDSSON, P. et J. WIKLUND (2001), «Levels of analysis in entrepreneurship research: current research practice and suggestions for the future», Entrepreneurship Theory and Practice, vol. 25, $\mathrm{n}^{\circ}$ 4, p. 81-99.

DE VILLERS, M.-É. (2009), Multidictionnaire de la langue française, 5édition, Montréal, Éditions Québec Amérique.

FILION, L.-J. (1997), «Le champ de l'entrepreneuriat: historique, évolution, tendances», Revue internationale PME, vol. 10, $\mathrm{n}^{\circ}$ 2, p. 129-172.

FORBES, D.F. (1999), «Cognitive approaches to new venture creation», International Journal of Management Reviews, vol. 1, n 4, p. 415-439.

Herron, L., H.J. SAPIENZA et D. SMITH-COOK (1991), «Entrepreneurship theory from an interdisciplinary perspective: volume I», Entrepreneurship Theory and Practice, vol. 16, $\mathrm{n}^{\circ}$ 2, p. 7-12.

Herron, L., H.J. SAPIENZA et D. SMITH-COOK (1992), «Entrepreneurship theory from an interdisciplinary perspective: volume II», Entrepreneurship Theory and Practice, vol. 16, n 3, p. 5-11.

HindLE, K. (2004), «Choosing qualitative methods for entrepreneurial cognition research: a canonical development approach», Entrepreneurship Theory and Practice, vol. 28, n 6, p. 575-607.

HofER, C.W. et W.D. BYGRAVE (1992), «Researching entrepreneurship», Entrepreneurship Theory and Practice, vol. 16, $\mathrm{n}^{\circ} 3$, p. 91-100.

LANGley, A. (1999), «Strategies for theorizing from process data», Academy of Management Review, vol. 24, nº 4, p. 691-710.

LOW, M.B. (2001), «The adolescence of entrepreneurship research: specification of purpose», Entrepreneurship Theory and Practice, vol. 25, n 4, p. 17-25.

LOW, M.B. et I.C. MACMiLLAN (1988), «Entrepreneurship: past research and future challenges », Journal of Management, vol. 14, n 2, p. 139-161.

MACMILlan, I.C. et J.A. KATZ (1992), «Idiosyncratic milieus of entrepreneurial research: the need for comprehensive theories », Journal of Business Venturing, vol. $7, \mathrm{n}^{\mathrm{o}} 1, \mathrm{p} .1-8$.

MeindL, J.R., C. StubbarT et J.F. PORAC (1994), «Cognition within and between organizations : five key questions », Organization Science, vol. 5, n 3, p. 289-293.

Mitchell, R.K., L. Busenitz, T. LANT, P.P. MCDougAll, E.A. MorSE et J.B. SMITH (2002), «Toward a theory of entrepreneurial cognition: rethinking the people side of entrepreneurship research», Entrepreneurship Theory and Practice, vol. 27, $\mathrm{n}^{\mathrm{o}} 2$, p. 93-104. 
Ouellet, C. et P. CossetTe (1999), «Les travaux des chercheurs utilisant le concept de cognition en sciences de l'administration: une étude exploratoire», Actes de la VIII Conférence internationale de management stratégique (AIMS), Paris.

PARTINGTON, D. et M. JenKins (2007), «Deconstructing scholarship: an analysis of research methods citations in the organizational sciences», Organizational Research Methods, vol. 10, no 3, p. 399-416.

SADLER-SMith, E. (2004), «Cognitive style and the management of small and mediumsized enterprises », Organization Studies, vol. 25, n 2, p. 155-181.

SCHNEIDER, S.C. et R. ANGELMAR (1993), «Cognition in organizational analysis: who's minding the store?», Organization Studies, vol. 14, n⿳ 3, p. 347-374.

ShANE, B. et S. VENKATARAMAN (2000), «The promise of entrepreneurship as a field of research », Academy of Management Review, vol. 25, n 1, p. 217-226.

STARBUCK, W.H. (1999), «Fussy Professor Starbuck's cookbook of handy-dandy prescriptions for ambitious academic authors or why I hate passive verbs and love my word processor », $<$ http://pages.stern.nyu.edu/ wstarbuc/Writing/Fussy. htm $>$.

Tenbrunsel, A.E., T.L. Galvin, M.A. Neale et M.H. BAZERman (1996), «Cognitions in organizations », dans S.R. Glegg, C. Hardy et W.N. Nord (dir.), Handbook of Organization Studies, Londres, Sage, p. 313-337.

UCBASARAN, D., P.WESTHEAD et M.WRIGHT (2001), «The focus of entrepreneurship research: contextual and process issues », Entrepreneurship Theory and Practice, vol. $25, \mathrm{n}^{\circ} 4$, p. 57-80.

Verstraete, T. et A. FAyOlle (2005), «Paradigmes et entrepreneuriat», Revue de l'entrepreneuriat, vol. 4, $\mathrm{n}^{\circ}$ 1, p. 33-52.

WALSH, J.P. (1995), «Managerial and organizational cognition: notes for a trip down memory lane », Organization Science, vol. 6, n 3, p. 280-321.

WeIcK, K.E. (1979), The Social Psychology of Organizing, 2 édition, New York, Random House.

WEICK, K.E. (1995), «Editing innovation into Administrative Science Quarterly», dans L.L. Cummings et P.J. Frost (dir.), Publishing in the Organizational Sciences, $2^{\mathrm{e}}$ édition, Thousand Oaks, Cal., Sage, p. 284-296.

WORTMAN, M.S. JR. (1987), «Entrepreneurship: an integrating typology and evaluation of the empirical research in the field», Journal of Management, vol. 13, $\mathrm{n}^{\circ} 2$, p. 259-279. 